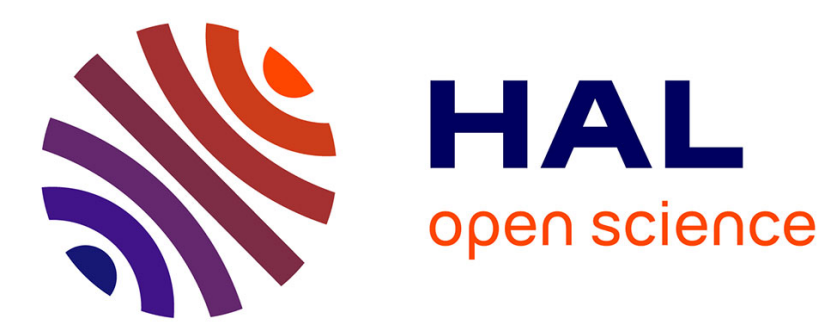

\title{
Room temperature magnetoelectric coupling in a molecular ferroelectric ytterbium(III) complex
}

Jérome Long, Maxim Ivanov, Vladimir Khomchenko, Ekaterina Mamontova, Jean-Marc Thibaud, Jérôme Rouquette, Mickael Beaudhuin, Dominique

Granier, Rute A.S. Ferreira, Luis Carlos, et al.

\section{To cite this version:}

Jérome Long, Maxim Ivanov, Vladimir Khomchenko, Ekaterina Mamontova, Jean-Marc Thibaud, et al. Room temperature magnetoelectric coupling in a molecular ferroelectric ytterbium(III) complex. Science, 2020, 367 (6478), pp.671-676. 10.1126/science.aaz2795 . hal-02470126

\section{HAL Id: hal-02470126 \\ https://hal.science/hal-02470126}

Submitted on 26 Nov 2020

HAL is a multi-disciplinary open access archive for the deposit and dissemination of scientific research documents, whether they are published or not. The documents may come from teaching and research institutions in France or abroad, or from public or private research centers.
L'archive ouverte pluridisciplinaire HAL, est destinée au dépôt et à la diffusion de documents scientifiques de niveau recherche, publiés ou non, émanant des établissements d'enseignement et de recherche français ou étrangers, des laboratoires publics ou privés. 


\section{Room temperature magnetoelectric coupling in a molecular ferroelectric Ytterbium(III) complex.}

J. Long $^{1 *}$, M. S. Ivanov², V. A. Khomchenko², E. Mamontova ${ }^{1}$, J.-M. Thibaud ${ }^{1}$, J. Rouquette $^{1}$, M. Beaudhuin ${ }^{1}$, D. Granier ${ }^{1}$, R. A. S. Ferreira ${ }^{3}$, L. D. Carlos ${ }^{3}$, B. Donnadieu ${ }^{4}$, M. S. C. Henriques ${ }^{2}$, J. A. Paixão ${ }^{2}, Y$. Guari ${ }^{1}$, J. Larionova $^{1}$

${ }^{1}$ Institut Charles Gerhardt Montpellier, UMR 5253, Université de Montpellier, ENSCM, CNRS, Place E. Bataillon, 34095 Montpellier Cedex, France.

${ }^{2}$ CFisUC, Department of Physics, University of Coimbra, 3004-516 Coimbra, Portugal.

${ }^{3}$ Physics Department and CICECO-Aveiro Institute of Materials, University of Aveiro, 3810-193, Aveiro, Portugal.

${ }^{4}$ Fédération de Recherche Chimie Balard-FR3105, Université de Montpellier, place E. Bataillon, 34095 Montpellier Cedex 5, France.

*Correspondence to: jerome.long@umontpellier.fr

Abstract: Magnetoelectrical (ME) materials combine magnetic and electric polarizabilities in the same phase. Such systems offer a basis for developing high-density data storage, spintronic or low consumption devices owing to the possibility to trigger one property by the other. Such objectives require a strong interaction between the constitutive properties, a criterion which is rarely met in classical inorganic ME materials at room temperature. We provide evidence of a strong $\mathrm{ME}$ coupling in a paramagnetic ferroelectric lanthanide coordination complex with magnetostrictive phenomenon. The properties of this molecular material show it may be competitive to inorganic magnetoelectrics.

One Sentence Summary: The room temperature magnetoelectric control of ferroelectric domains was demonstrated in a $\mathrm{Yb}^{3+}$ molecule-based material.

A ferroelectric material exhibits a permanent electrical polarization that can be switched by an electric field $(1,2)$. Such electro-active materials find a wide range of applications including temperature sensing, data-storage, piezoelectric devices, and electro-optics. The association of electrical and magnetic polarizabilities gives rise to exciting multifunctional systems denoted as magnetoelectrics (ME) which describes the influence of a magnetic (electric) field on the polarization (magnetization) allowing the constitutive properties to be simultaneously triggered $(3,4)$. For computing, modifying the polarization/magnetization using low-magnitude magnetic/electric field may reduce the energy needed and speed up non-volatile memory devices' processing's rate $(3,5,6)$. Conventional approaches to designing single-phase ME are widely based on inorganic materials, such as oxides or fluorides (6-8). However, if the origins of ferroelectricity and magnetism are associated with different carriers, we should expect only a moderate ME coupling (9). For instance, only a few examples exist of ME coupling at room temperature $(6,10,11)$. In contrast, large $\mathrm{ME}$ coupling has been found in strain mediated multi-phase materials, such as composites or multi-layers, by combining piezoelectricity with the magnetostrictive effect (3).

Molecular materials exhibit numerous advantages in comparison to inorganic ones, such as structural diversity, soft chemistry routes, environmentally friendly processing and shaping, optical transparency, and light density (12). For these reasons, molecular ferroelectrics $(13,14)$ are often considered as an alternative towards traditional metal oxides 
$(15,16)$. Even though molecular materials exhibit magnetic ordering temperature below room temperature, important ME coupling may be expected by the association of paramagnetism and ferroelectricity (Fig. S1) (7) since both these properties could involve the same chemical element. Although ME coupling in non-ferroelectric molecular materials has been reported $(17,18)$, the interaction between magnetism and ferroelectricity is not extensively studied (19-26). The ferroelectric ordering temperatures are typically lower than room temperature for the few examples that exist. Moreover, the modification of the polarization/magnetization by applying magnetic/electric fields, which remains also challenging in pure inorganic materials, requires large magnitude operating magnetic field (27).

10 We designed a chiral lanthanide complex exhibiting an above-room temperature ferroelectricity which, in association with a strong magnetostriction, gives rise to a remarkable ME coupling. This allowed tuning of the ferroelectric domains at the nanometric scale by applying a relatively low magnetic field at room temperature. Our molecular approach to design ME materials relied on the association of paramagnetic lanthanide ions, such as $\mathrm{Yb}^{3+}$, with a chiral diamagnetic zinc complex in order to favor the crystallization in one of the ten polar point groups compatible with ferroelectricity. We chose the $\mathrm{Yb}^{3+}$ ion because it possesses a large total magnetic moment, which being oriented along a magnetic field can provide an anisotropic magnetostriction underlying the coupling between magnetic and structural subsystems.

20 The stoichiometric reaction of $R, R-\mathrm{H}_{2} \mathrm{~L} \quad\left(2,2^{\prime}-[[(1 \mathrm{R}, 2 \mathrm{R})-1,2-\right.$ diphenyl-1,2ethanediyl]bis[(E)-nitrilomethylidyne]]bis[6-methoxy) or $\quad S, S-\mathrm{H}_{2} \mathrm{~L} \quad\left(2,2^{\prime}-[[(1 \mathrm{R}, 2 \mathrm{R})-1,2-\right.$ diphenyl-1,2-ethanediyl]bis[(E)- nitrilomethylidyne]]bis[6-methoxy), $\mathrm{Zn}(\mathrm{OAc})_{2} \cdot 2 \mathrm{H}_{2} \mathrm{O}$ and $\mathrm{Yb}\left(\mathrm{NO}_{3}\right)_{3} \cdot 5 \mathrm{H}_{2} \mathrm{O}$ in methanol yielded to a yellow solution which upon slow diffusion of diethylether resulted in the crystallization of $R, R-\left[\mathrm{Zn}(\mathrm{OAc})(\mathrm{L}) \mathrm{Yb}\left(\mathrm{NO}_{3}\right)_{2}\right](R, R-\mathbf{1})$ or $S, S$ $\left[\mathrm{Zn}(\mathrm{OAc})(\mathrm{L}) \mathrm{Yb}\left(\mathrm{NO}_{3}\right)_{2}\right](S, S-2)$. We found using single crystal X-ray diffraction that $R, R-\mathbf{1}$ and $S, S-2$ are isostructural to the dysprosium analogue (28). The two enantiomers crystallized in the polar space group $P 2{ }_{1}$ with two independent homochiral dinuclear complexes within the asymmetric unit (Fig. 1, Fig. S2-S3). These two complexes exhibit differences in the crystallographic distances and geometries (Tables S1-S2) that gives rise to uncompensated dipolar moments along the $b$ axis. We confirmed this by analyzing the ions' location in the unit cell (Table S3)(29-31).

We corroborated the enantiomeric nature of $R, R-\mathbf{1}$ and $S, S-2$ using solid state circular dichroism (CD) (Fig. S4), with the mirror-symmetrical CD spectra showing Cotton effects of opposite signs at $\lambda_{\max }=261,303$ and $386 \mathrm{~nm}$. The presence of the zinc complex acted also as a sensitizer towards lanthanide ions. Hence, the complexes $R, R-\mathbf{1}$ and $S, S-2$ showed the typical $\mathrm{Yb}^{3+}$ luminescence in the $925-1075 \mathrm{~nm}$ NIR region at both, room and low temperatures (Fig. S5-S11(32)). 


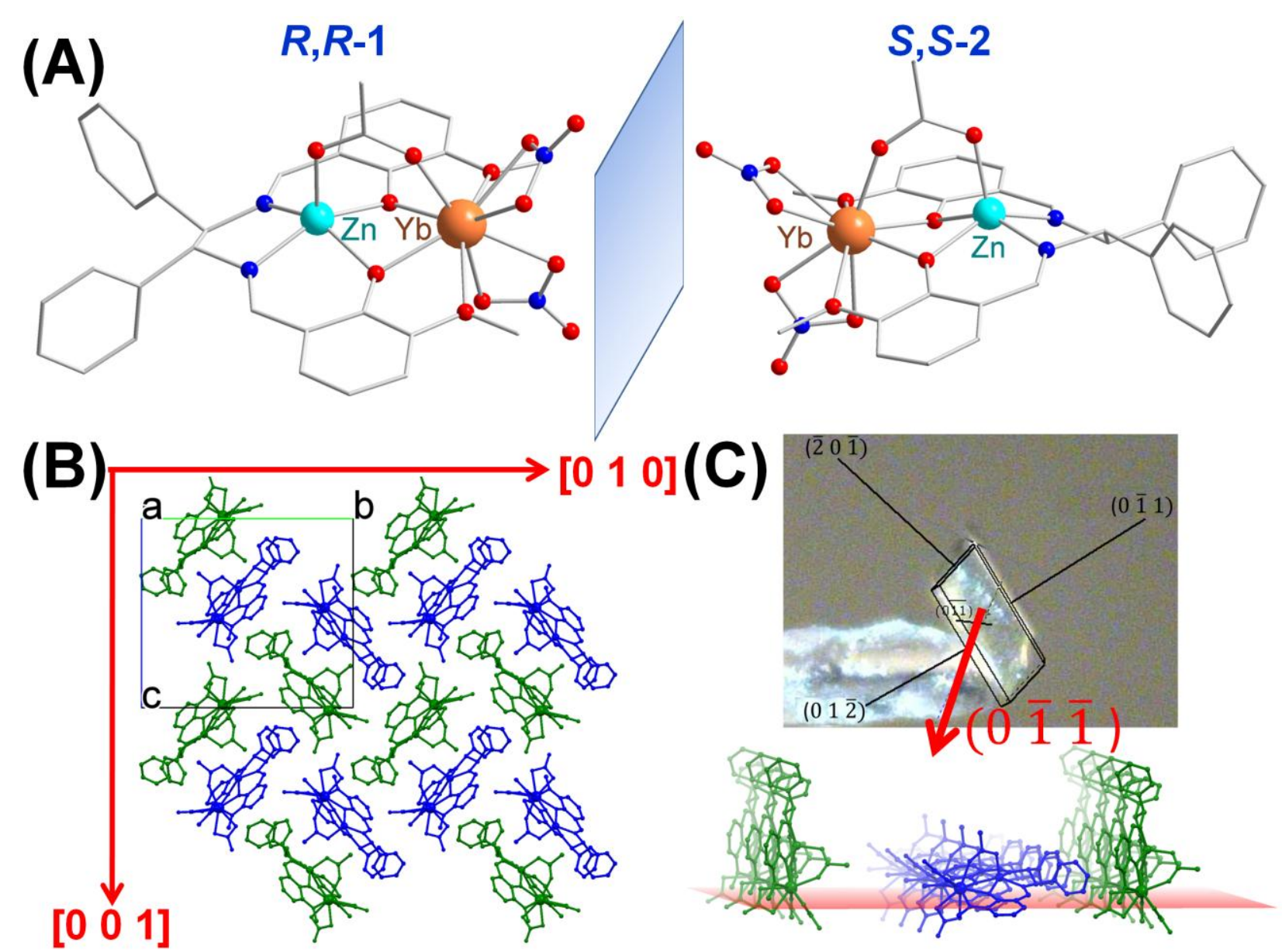

Fig. 1. Crystal structures of $R, R-1$ and $S, S-2$. (A) Molecular structure of the dinuclear $\mathrm{Zn}^{2+} / \mathrm{Yb}^{3+}$ complexes $R, R-\mathbf{1}$ and $S, S-2$ and their enantiomeric relationship. Color code: orange $\mathrm{Yb}^{3+}$, light blue $\mathrm{Zn}^{2+}$, blue $\mathrm{N}$, red $\mathrm{O}$, grey $\mathrm{C}$. Hydrogen atoms have been omitted for clarity. (B) View of the crystal packing arrangement of $R, R-\mathbf{1}$ along the $a$ axis emphasizing the two homochiral complexes. (C) Single-crystal facets assignment and view of the slice in the crystallographic (0 $\overline{1} \overline{1})$ plane.

We verified the absence of solvent molecules with thermogravimetric analyzes (TGA) which also indicated that both enantiomers remain stable up to $550 \mathrm{~K}$ (Fig. S12). We also used single X-ray diffraction at $400 \mathrm{~K}$ and differential scanning calorimetry (DSC) to confirm the absence of a phase transition up to the decomposition temperature (Fig. S13). This demonstrated that the material remains crystallized in the $P 2_{1}$ polar space group. We investigated the magnetic properties of $R, R-\mathbf{1}$ and $S, S-2$ using SQUID magnetometry. We confirmed the paramagnetic behavior expected for a single $\mathrm{Yb}^{3+}$ ion with a ${ }^{2} \mathrm{~F}_{7 / 2}$ ground state $(J=7 / 2, L=3, S=1 / 2)$ and a room temperature magnetic moment close to $4.32 \mu_{\mathrm{B}}$ (Fig. S14). We also observed a typical slow relaxation of the magnetization at low temperature (Fig. S15-S20, Tables S4-S6(32)).

Both enantiomers crystallized in the acentric space group $P 2{ }_{1}$, giving a 2 point group. We confirmed ferroelectric behavior of $R, R-1$ (Fig. S21-S22(32)) which also applies to $S, S-2$ because the ferroelectric properties of pairs of enantiomers are known to be identical $(33,34)$. The absence of phase transition up to the decomposition of the material $(550 \mathrm{~K})$ indicates that the ferroelectric Curie point $\left(T_{\mathrm{c}}\right)$ shall be located at a higher temperature as observed, for instance, in such ferroelectrics as $\left[\left(\mathrm{CH}_{3}\right)_{4} \mathrm{~N}\right] \mathrm{HgCl}_{3}$ or $\left[\mathrm{C}\left(\mathrm{NH}_{2}\right)_{3}\right]\left[\mathrm{Al}\left(\mathrm{H}_{2} \mathrm{O}\right)_{6}\right]\left(\mathrm{SO}_{4}\right)_{2}(14)$. 
(A)
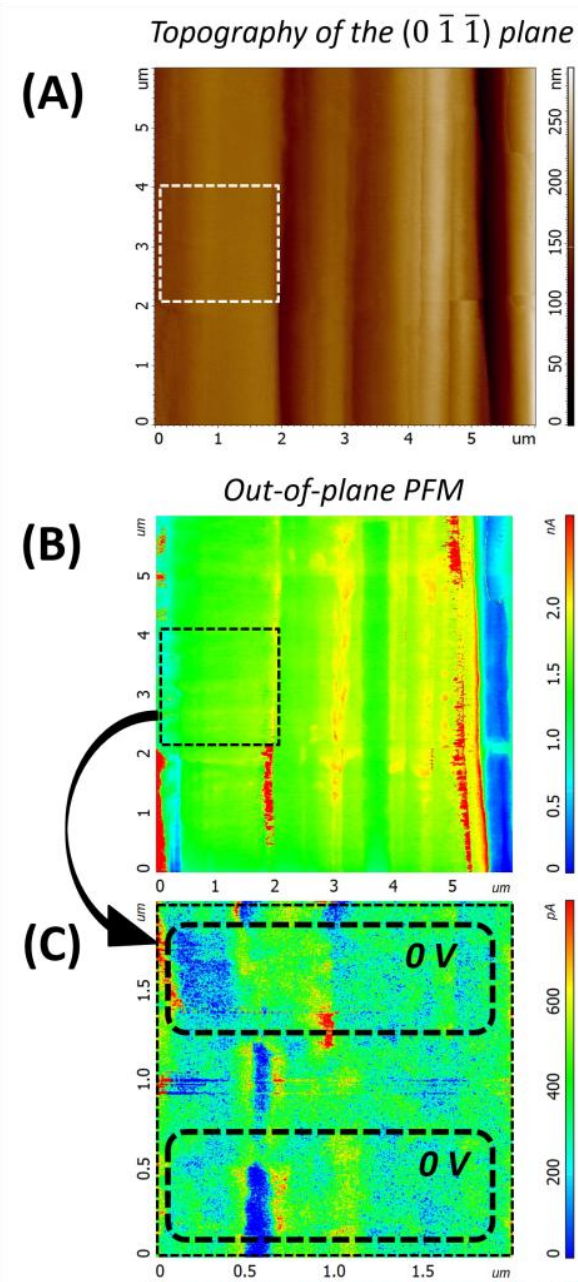

(D)

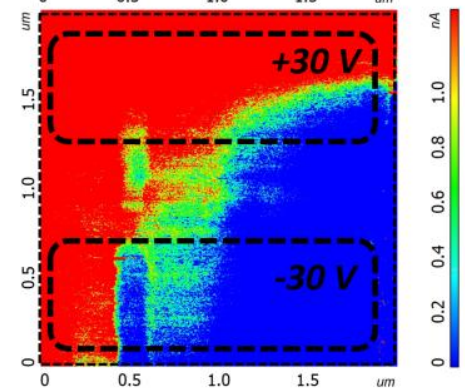

(E)

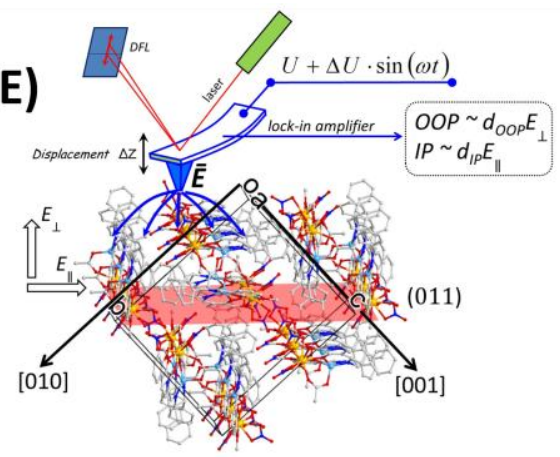

In-plane PFM
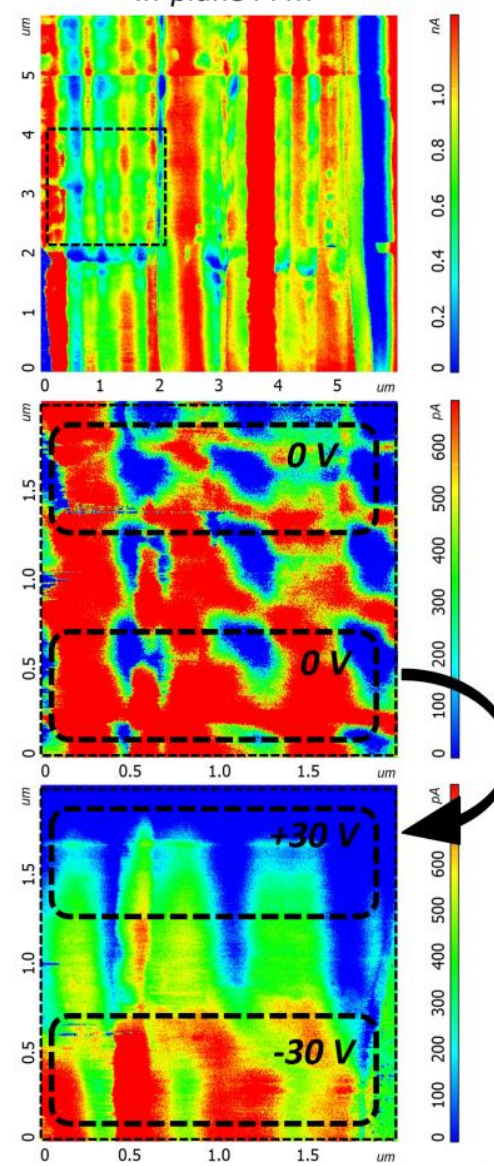

Fig. 2. Scanning probe microscopy (SPM) measurements of the $(0 \overline{\mathbf{1}} \overline{\mathbf{1}})$-oriented $R, R-1$ single crystal: (A) AFM topography of the ( $0 \overline{1} \overline{1})$ plane. (B) OOP (left) and IP (right) PFM responses without electrical bias. The color mapping reveals a peculiar organization demonstrating the presence of spontaneously polarized domains of opposite polarization states (shown in red and blue). (C) Magnified $(\times 3)$ part of the scanned area (marked as a square in the previous images) with the OOP (left) and IP (right) PFM responses at $0 \mathrm{~V}$. (D) OOP (left) and IP (right) PFM responses measured for the same area after the direct current $(d c)$ bias voltage $( \pm 30 \mathrm{~V})$ poling. (E) Schematic of the PFM experiment.

We carried out piezoresponse force microscopy (PFM) measurements to investigate the polar behavior and ME coupling $(32,35)$. We measured both the vertical and lateral responses, each of them consisted of amplitude and phase signals directly related to the magnitude of the polarization and to its orientation, respectively. This method provided sufficient spatial resolution for the detailed study of in-plane (IP, lateral PFM amplitude $\times$ 
phase) and out-of-plane (OOP, vertical PFM amplitude $\times$ phase) piezoelectric behavior at the nanoscale $(35,36)$. Because additional contributions having ionic transport or electrostatic nature could affect the PFM signals, we carried out the measurements taking into account the protocol described by Vaseduvan et al. (37) and Balke et al. (38) (Fig. S23-S24,(32)). Hence, the PFM responses we observed reflected the polarization state of the material. We obtained a clear piezoresponse coming from several planes of $R, R-\mathbf{1}$ single crystal (Fig. S25), but we detected the strongest simultaneous OOP and IP responses for the largest crystal facet accounting for the $(0 \overline{1} \overline{1})$ plane (Fig. S26). The atomic force microscopy (AFM) for the $(0 \overline{1} \overline{1})$ plane reveals a stripe-like morphology (Fig. 2A). We found a peculiar organization in the OOP and IP PFM response that demonstrated the presence of spontaneously polarized domains of opposite polarization states (shown in red and blue, Fig. 2B). Such stripes organization was previously observed in others molecular or metal oxides ferroelectrics such as $\mathrm{BiFeO}_{3}(35,39-41)$. The crystal symmetry predicts that the ferroelectric domain structure visualized by the IP and OOP components of the piezoresponse must be consistent. However, the resulting PFM response depends on the azimuthal angle between the scanning direction and the polar axis and contains different tensile and shear piezoelectric strain components (as described in the "PFM Measurements" subsection of the Supplementary Materials). This situation is reminiscent to that observed in the benchmark molecular ferroelectric diisopropylammonium bromide (40). The number of polarization directions in the ferroelectric phase depends on the symmetry of the paraelectric phase according to the initial work of Aizu (42) and recently applied to molecular ferroelectrics $(13,43,44)$. Although the structure of the paraelectric phase cannot be examined $\left(T_{\mathrm{c}}\right.$ is higher than decomposition temperature), thus precluding the determination of the uniaxial or multiaxial character of the polarization, clear correlation between the IP and OOP responses can be observed in some other PFM experiments (Fig. S27).

We investigated the polarization switchability using direct current $(d c)$ bias voltage of $\pm 30 \mathrm{~V}$ applied to different areas (rectangulars in Fig. 2C, D). Within these areas, we achieved uniform (monodomain-like) polarization states, visible on both, IP and OOP images (Fig. 2D). Taking advantage of the absence of a phase transition, we performed PFM measurements at $450 \mathrm{~K}$ (Fig. S24(32)). We found a notable decrease in the electromechanical response, directly related to the polarization, which reflected the temperature dependent behavior for a ferroelectric material. The switchable character of the electric polarization was further demonstrated by applying opposite dc bias to generate box-in-box patterns that showed clear $180^{\circ}$ phase contrast and domain walls (Fig. S28). Moreover, the piezoresponse hysteresis loops obtained by switching spectroscopy (SS)-PFM was found to exhibit a centered square-like shape with a $180^{\circ}$ switching for the phase component (Fig. 3A). We also measured the typical butterfly loops of the displacement signal which confirmed the ferroelectric character (37). Previously switched areas $( \pm 30 \mathrm{~V})$ revealed a shift of the phase and amplitude component towards either positive or negative voltage, due to the formation of coherent remnant polarization states that caused a strong depolarization field (Fig. 3A). We determined a maximum local longitudinal piezoelectric coefficient $\mathrm{d}_{\mathrm{OOP}}^{*}=73 \mathrm{pm} . \mathrm{V}^{-1}$ at room temperature, which corresponded to a polarization of the order of magnitude of 10 $\mu \mathrm{C} . \mathrm{cm}^{-2}$. The theoretical spontaneous polarization which we estimated from the point charge model (29-31) along the $b$ axis by considering only the metal ions and some ligand's atoms $\left(\mathrm{Zn}^{2+}, \mathrm{Yb}^{3+}, \mathrm{O}^{-}\right.$and $\mathrm{N}^{+}$) gives $3.32 \mu \mathrm{C} . \mathrm{cm}^{-2}$ along the $\left[\begin{array}{lll}0 & \overline{1} & \overline{1}\end{array}\right]$ direction (see Table S3 (32)). We can rationalize this value which was weaker than the experimental one, by pointing out the complexity of the structure. Besides, we did not take into account the effect of additional molecular dipoles constituting the coordination complexes as well as covalency. Thus, we could compare the experimental value with Rochelle salt $\left(0.25 \mu \mathrm{C} . \mathrm{cm}^{-2}\right)$ and was the same order of magnitude that the better performing molecular ferroelectrics $(14,15,40,41)$. 

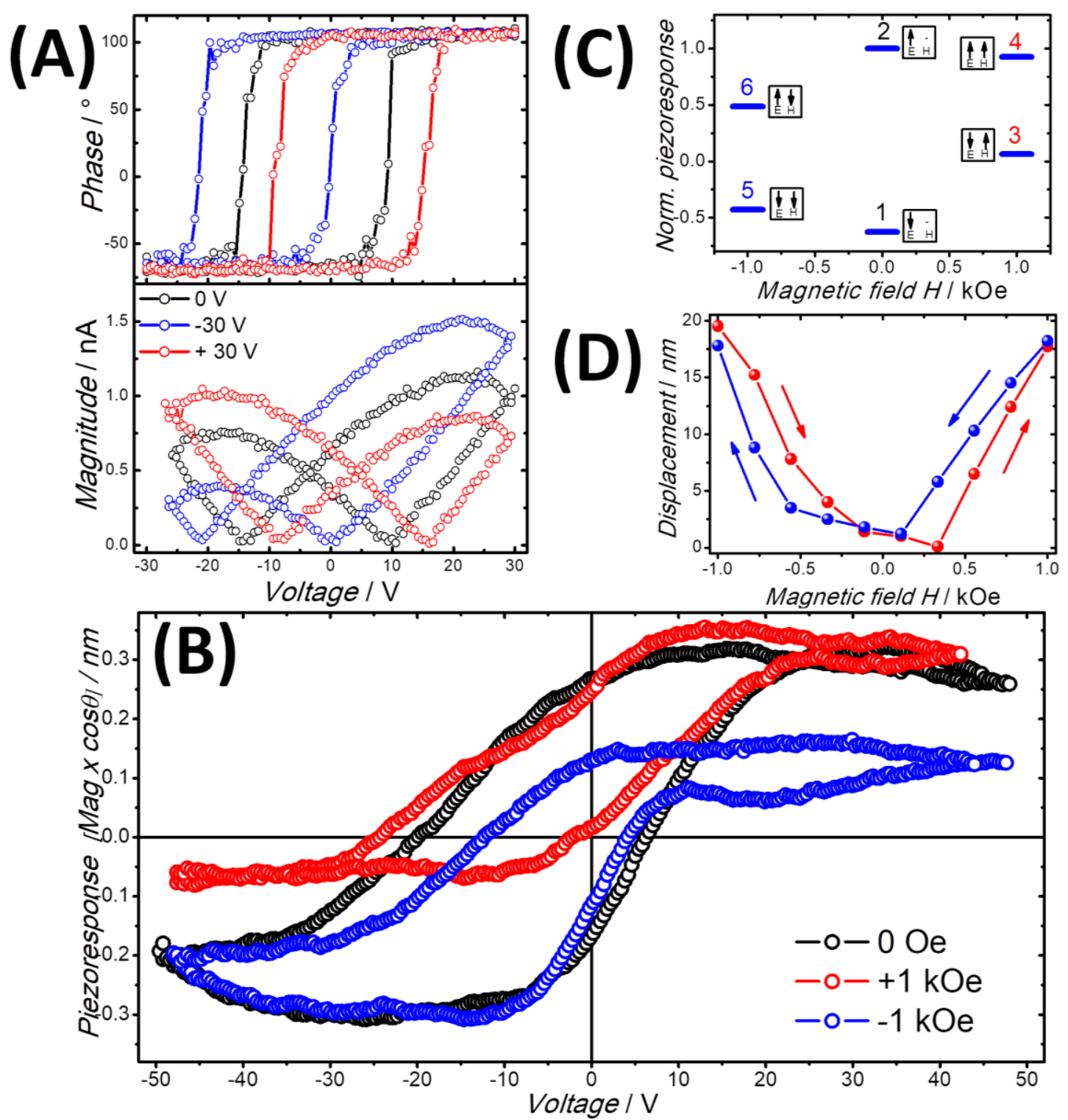

Fig. 3. SS-PFM hysteresis loops, multilevel states and magnetostriction. (A) SS-PFM hysteresis loops obtained for the virgin $(0 \mathrm{~V})$ and polished by $d c$ bias voltage $( \pm 30 \mathrm{~V})$ areas: phase and displacement components as a function of the voltage. (B) SS-PFM hysteresis loops obtained at zero and under applied magnetic field of $\pm 1 \mathrm{kOe}$. (C) Normalized piezoresponse as a function of the magnetic field enlightening the six remanent polarization states that could be actuated by applying magnetic and/or electric fields. (D) Magnetostriction measured on a single crystal of $R, R-\mathbf{1}$ at $\pm 1 \mathrm{kOe}$ (averaged on 5 loops). The lines are guide for the eye.

The ferroelectric character of $R, R-\mathbf{1}$ crystals suggests to us the possibility of a ME coupling at room temperature. We performed PFM measurements on the same single crystal in the presence of a dc magnetic field of $\pm 1 \mathrm{kOe}$ applied along the $\left(\begin{array}{ll}0 & \overline{1} \\ \overline{1}\end{array}\right)$ plane. We observed the stripe-like morphology obtained by AFM on a new surface area (Fig. 4A). We found ferroelectric polarization redistribution with a low magnitude magnetic field of $\pm 1 \mathrm{kOe}$, similar to that produced by an electrical bias voltage (Fig. 4B, 4C). The change of the polarization states and enhancement of the response make the effect easy to see. The 
ferroelectric domain's modification appears only in some parts of the region as it has been systematically observed in the rare examples of materials investigated by PFM (27, 45-47). The typical magnitude of the magnetoelectric tensor component $\alpha_{31}=\frac{\Delta \mathrm{u}}{\mathrm{d}_{\mathrm{oop}} \Delta H D}$ where $D$ is the thickness of the $(0 \overline{1} \overline{1})$-oriented crystal, $\Delta u$ is the change in vertical surface displacement induced by the change in lateral magnetic field $\Delta H$, attains the order of $100 \mathrm{mV} . \mathrm{Oe}^{-1} . \mathrm{cm}^{-1}$. Although this value reflects the polarization change for a given magnetic field, it greatly exceeded (by at least one order magnitude) those observed in the bulk $\mathrm{BiFeO}_{3}$ multiferroics (ranging from 0.6 to $7 \mathrm{mV} \cdot \mathrm{Oe}^{-1} \cdot \mathrm{cm}^{-1}$ ) (48) and was comparable with those of ferroelectric/ferro(ferri)magnetic composites with strain-mediated ME coupling (49). Additional confirmation of the change in responses comes from our in-situ PFM measurements performed on other crystal facets and with various magnetic fields (Fig. S29S32(32)).

We found additional evidence of the ME interaction from our SS-PFM local hysteresis loops measurements we performed under dc magnetic field. These loops were strongly affected by a magnetic field, specifically in their asymmetry, height, and in the coercive fields (Fig. 3B). Taking advantage of such ME coupling, we actuated variable polarization states via the dc electric and magnetic fields (Fig. 3C). Such feature may be suitable for multilevel polarization states devices designed for high-density data systems (50). Remarkably, this interaction occurs in the paramagnetic state at room temperature using a moderate magnetic field $(1 \mathrm{kOe})$. The low magnetic field we used clearly contrasts with other molecule-based materials that required fields of several Tesla to induce a change in the pyroelectric currents (23). Such room-temperature low-field switching is also quite rare in metal oxides $(11,26,27)$.

The strong ME effect we observed is because the same chemical element, $\mathrm{Yb}^{3+}$, is implicated in the two functionalities. Lanthanide ions present a larger spin-orbit coupling with respect to transition metal ions in classical inorganic magnetic ferroelectrics. Applying a magnetic field to the material containing anisotropic $\mathrm{Yb}^{3+}$ (non-zero orbital angular momentum, $L=3$, spin-orbit coupling) should affect the crystal lattice producing magnetostriction. To confirm this, we investigated the magnetostriction, $\lambda$, at the microscopic and macroscopic levels. We measured the local surface displacement using contact AFM mode (no electric field applied) and evaluated it as a function of the applied magnetic field. We found a large room-temperature field-induced mechanical deformation (parastriction) (Fig. 3D). The displacement increased with magnetic field and reached about $10^{-4}(1 \mathrm{kOe})$. The magnetostriction value we found was consistent with the macroscopic data we obtained from single-crystal X-ray diffraction experiment we conducted under a magnetic field of about $0.8 \mathrm{kOe}$ applied with a deviation angle of $10 \pm 5^{\circ}$ along the $\left[\begin{array}{lll}0 & 1 & \overline{1}\end{array}\right]$ direction and yielding $\lambda$ value of up to $1 \times 10^{-3}$ (Table S7(32)). We confirmed this effect on a different single-crystal and by collecting three data sets to provide a statistical analysis (Table S8). Reversing the direction of the magnetic field did not change the sign of the magnetostriction (Table S7), which was fully consistent with the expected quadratic behavior. This large magnetostriction was comparable to those observed in ytterbium-based paramagnets. This phenomenon had a single-ion character primarily, and the related deformation can be equivalent in magnitude with that characteristic of magnetically long-range ordered systems (51). Importantly, the association of magnetostriction and ferroelectricity engenders a pronounced ME coupling as observed in inorganic multi-phase ME materials (3). 

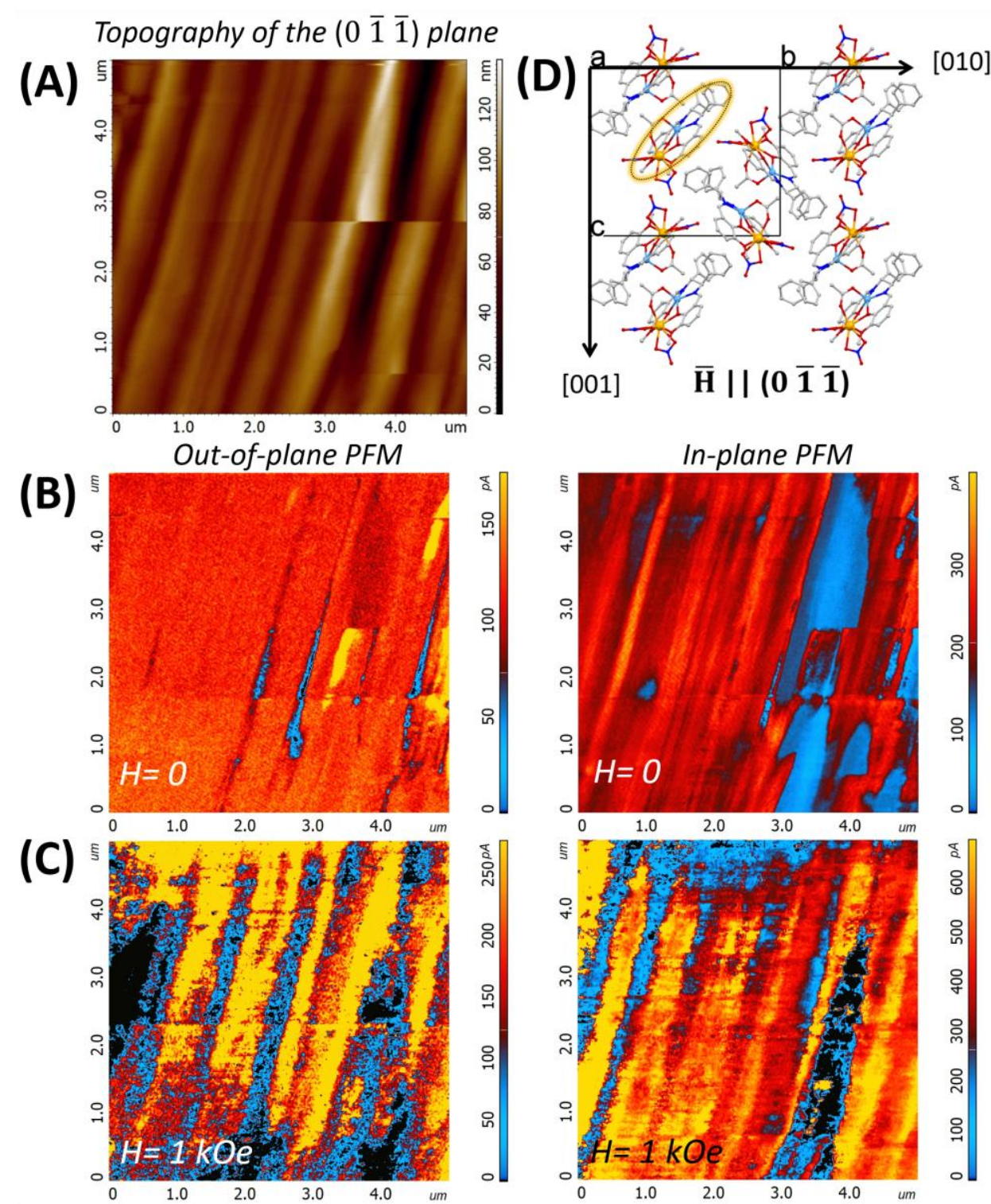

Fig. 4. Room temperature PFM studies under magnetic field evidencing the ME coupling. (A) AFM topography of the $(0 \overline{1} \overline{1})$-plane for $R, R-\mathbf{1}$. (B) OOP and IP PFM responses at $H=0$ Oe. (C) OOP and IP PFM responses at $H=1 \mathrm{kOe}$ evidencing the ME coupling as redistribution in the ferroelectric domains (change in colors) and increase in the electromechanical response. (D) Sketch illustrating the possible deformation of the individual complex under an applied magnetic field.

Thus, we propose that the ME interaction in our $\mathrm{Yb}^{3+}$-based ferroelectric complex originates from magnetoelastic coupling that corresponds to the magnetic field-induced deformation of the crystal lattice in the paramagnetic phase. Because $R, R-\mathbf{1}$ exhibited simultaneously magnetostriction and ferroelectricity, applying a magnetic-field induced a mechanical strain via spin-lattice coupling that in turn influenced the polarization by affecting the overall dipole configuration. To support this, we made a comparative analysis of the crystal structures we obtained under two directions of the magnetic field with respect to the zero-field one. Differences in the metal-ligand distances (Table S9 and S10) for both $\mathrm{Yb}^{3+}$ and $\mathrm{Zn}^{2+}$ ions could be discerned that in turn affect the dipole order. Hence, the polarization values we calculated were found up to $10 \%$ greater or weaker (Table S3 and S11), depending 
on the orientation of the magnetic field, with respect to the zero-magnetic field value. Such results are in line with those obtained by PFM.

We have demonstrated room temperature magnetoelectric control of ferroelectric domains in a molecule-based material. Thus, in the $\mathrm{Yb}^{3+}$-based chiral compound $R, R-\mathbf{1}$, the combination of ferroelectric behavior with a magnetostrictive effect generates a strong ME coupling we observed at room temperature and with a relatively low magnetic field. These properties are useful for practical device application including non-volatile memory where information would be stored as electrically detectable and controllable by $\mathrm{Yb}^{3+}$ paramagnetism. More generally, such features appear particularly unique in single-phase materials and confirm that the genuine chemical design of multifunctional molecular materials may provide an alternative strategy to usual solid-state compounds for engineering ME devices.

1. S.-T. Han, Y. Zhou, V. A. L. Roy, Towards the Development of Flexible NonVolatile Memories. Adv. Mater. 25, 5425-5449 (2013)10.1002/adma.201301361).

2. S. Hong, O. Auciello, D. Wouters, Emerging Non-Volatile Memories. (Springer US, 2014).

3. W. Eerenstein, N. D. Mathur, J. F. Scott, Multiferroic and magnetoelectric materials. Nature 442, 759-765 (2006); published online Epub08/17/print (

4. K. F. Wang, J. M. Liu, Z. F. Ren, Multiferroicity: the coupling between magnetic and polarization orders. Adv. Phys. 58, 321-448 (2009); published online Epub2009/07/01 (10.1080/00018730902920554).

5. J. F. Scott, Data storage: Multiferroic memories. Nat. Mater. 6, 256-257 (2007); published online Epub04//print (

6. P. Mandal, M. J. Pitcher, J. Alaria, H. Niu, P. Borisov, P. Stamenov, J. B. Claridge, M. J. Rosseinsky, Designing switchable polarization and magnetization at room temperature in an oxide. Nature 525, 363-366 (2015); published online Epub09/17/print (10.1038/nature14881).

7. M. Fiebig, T. Lottermoser, D. Meier, M. Trassin, The evolution of multiferroics. Nature Reviews Materials 1, 16046 (2016); published online Epub07/05/online (10.1038/natrevmats.2016.46).

8. J. F. Scott, R. Blinc, Multiferroic magnetoelectric fluorides: why are there so many magnetic ferroelectrics? J. Phys.: Condens. Matter 23, 113202 (2011).

9. S.-W. Cheong, M. Mostovoy, Multiferroics: a magnetic twist for ferroelectricity. Nat. Mater. 6, 13-20 (2007); published online Epub01//print (

10. M. J. Pitcher, P. Mandal, M. S. Dyer, J. Alaria, P. Borisov, H. Niu, J. B. Claridge, M. J. Rosseinsky, Tilt engineering of spontaneous polarization and magnetization above $300 \mathrm{~K}$ in a bulk layered perovskite. Science 347, 420-424 (2015)10.1126/science.1262118).

11. Y. Kitagawa, Y. Hiraoka, T. Honda, T. Ishikura, H. Nakamura, T. Kimura, Low-field magnetoelectric effect at room temperature. Nat. Mater. 9, 797-802 (2010); published online Epub10//print (

12. D. W. Bruce, D. O'Hare, R. I. Walton, Molecular Materials. (Wiley, 2011).

13. T. Hang, W. Zhang, H.-Y. Ye, R.-G. Xiong, Metal-organic complex ferroelectrics. Chem. Soc. Rev. 40, 3577-3598 (2011)10.1039/C0CS00226G). 
14. W. Zhang, R.-G. Xiong, Ferroelectric Metal-Organic Frameworks. Chem. Rev. 112, 1163-1195 (2012); published online Epub2012/02/08 (10.1021/cr200174w).

15. S. Horiuchi, Y. Tokunaga, G. Giovannetti, S. Picozzi, H. Itoh, R. Shimano, R. Kumai, Y. Tokura, Above-room-temperature ferroelectricity in a single-component molecular crystal. Nature (London, U. K.) 463, 789-792 (2010); published online Epub// (10.1038/nature08731).

16. S. Horiuchi, Y. Tokura, Organic ferroelectrics. Nat. Mater. 7, 357 (2008); published online Epub05/01/online (10.1038/nmat2137).

17. A. K. Boudalis, J. Robert, P. Turek, First Demonstration of Magnetoelectric Coupling in a Polynuclear Molecular Nanomagnet: Single-Crystal EPR Studies of [Fe3O(O2CPh)6(py)3]ClO4.py under Static Electric Fields. Chem. Eur. J. 24, 1489614900 (2018); published online Epub2018/10/09 (10.1002/chem.201803038).

18. M. Fittipaldi, A. Cini, G. Annino, A. Vindigni, A. Caneschi, R. Sessoli, Electric field modulation of magnetic exchange in molecular helices. Nat. Mater. 18, 329-334 (2019); published online Epub2019/04/01 (10.1038/s41563-019-0288-5).

19. P. Jain, V. Ramachandran, R. J. Clark, H. D. Zhou, B. H. Toby, N. S. Dalal, H. W. Kroto, A. K. Cheetham, Multiferroic Behavior Associated with an Order-Disorder Hydrogen Bonding Transition in Metal-Organic Frameworks (MOFs) with the Perovskite ABX3 Architecture. J. Am. Chem. Soc. 131, 13625-13627 (2009); published online Epub2009/09/30 (10.1021/ja904156s).

20. F. Kagawa, S. Horiuchi, M. Tokunaga, J. Fujioka, Y. Tokura, Ferroelectricity in a one-dimensional organic quantum magnet. Nat. Phys. 6, 169-172 (2010); published online Epub03//print (http://www.nature.com/nphys/journal/v6/n3/suppinfo/nphys1503_S1.html).

21. G.-C. Xu, W. Zhang, X.-M. Ma, Y.-H. Chen, L. Zhang, H.-L. Cai, Z.-M. Wang, R.-G. Xiong, S. Gao, Coexistence of Magnetic and Electric Orderings in the Metal-Formate Frameworks of [NH4][M(HCOO)3]. J. Am. Chem. Soc. 133, 14948-14951 (2011); published online Epub2011/09/28 (10.1021/ja206891q).

22. H.-L. Cai, Y. Zhang, D.-W. Fu, W. Zhang, T. Liu, H. Yoshikawa, K. Awaga, R.-G. Xiong, Above-Room-Temperature Magnetodielectric Coupling in a Possible Molecule-Based Multiferroic: Triethylmethylammonium Tetrabromoferrate(III). $J$. Am. Chem. Soc. 134, 18487-18490 (2012); published online Epub2012/11/14 (10.1021/ja3073319).

23. W. Wang, L. Q. Yan, J. Z. Cong, Y. L. Zhao, F. Wang, S. P. Shen, T. Zou, D. Zhang, S. G. Wang, X. F. Han, Y. Sun, Magnetoelectric coupling in the paramagnetic state of a metal-organic framework. Sci. Rep. 3, 2024 (2013); published online Epub06/19/online (10.1038/srep02024 http://www.nature.com/articles/srep02024\#supplementary-information).

24. J. Alberto Rodríguez-Velamazán, Ó. Fabelo, Á. Millán, J. Campo, R. D. Johnson, L. Chapon, Magnetically-induced ferroelectricity in the (ND4)2[FeCl5(D2O)] molecular compound. Sci. Rep. 5, 14475 (2015); published online Epub09/29/online (10.1038/srep14475 https://www.nature.com/articles/srep14475\#supplementaryinformation).

25. M. Naka, S. Ishihara, Magnetoelectric effect in organic molecular solids. Sci. Rep. 6, 20781 (2016); published online Epub02/15/online (10.1038/srep20781 https://www.nature.com/articles/srep20781\#supplementary-information).

26. H. Ueda, T. Akita, Y. Uchida, T. Kimura, Room-temperature magnetoelectric effect in a chiral smectic liquid crystal. Appl. Phys. Lett. 111, 262901 (2017); published online Epub2017/12/25 (10.1063/1.5007317). 
27. D. M. Evans, A. Schilling, A. Kumar, D. Sanchez, N. Ortega, M. Arredondo, R. S. Katiyar, J. M. Gregg, J. F. Scott, Magnetic switching of ferroelectric domains at room temperature in multiferroic PZTFT. Nat. Commun. 4, 1534 (2013); published online Epub02/26/online (10.1038/ncomms2548 https://www.nature.com/articles/ncomms2548\#supplementary-information).

28. J. Long, J. Rouquette, J.-M. Thibaud, R. A. S. Ferreira, L. D. Carlos, B. Donnadieu, V. Vieru, L. F. Chibotaru, L. Konczewicz, J. Haines, Y. Guari, J. Larionova, A HighTemperature Molecular Ferroelectric Zn/Dy Complex Exhibiting Single-Ion-Magnet Behavior and Lanthanide Luminescence. Angew. Chem., Int. Ed. 54, 2236-2240 (2015); published online Epub// (10.1002/anie.201410523).

29. P.-F. Li, Y.-Y. Tang, Z.-X. Wang, H.-Y. Ye, Y.-M. You, R.-G. Xiong, Anomalously rotary polarization discovered in homochiral organic ferroelectrics. Nat. Comm. 7, 13635 (2016); published online Epub11/23/online (10.1038/ncomms13635 https://www.nature.com/articles/ncomms13635\#supplementary-information).

30. Y.-M. You, Y.-Y. Tang, P.-F. Li, H.-Y. Zhang, W.-Y. Zhang, Y. Zhang, H.-Y. Ye, T. Nakamura, R.-G. Xiong, Quinuclidinium salt ferroelectric thin-film with duodecuplerotational polarization-directions. Nat. Comm. 8, 14934 (2017); published online Epub04/04/online (10.1038/ncomms 14934 https://www.nature.com/articles/ncomms14934\#supplementary-information). R.-G. Xiong, Directional Intermolecular Interactions for Precise Molecular Design of a High-Tc Multiaxial Molecular Ferroelectric. J. Am. Chem. Soc. 141, 1781-1787 (2019); published online Epub2019/01/30 (10.1021/jacs.8b13223).

32. Materials and Methods are available as Supplementary Materials on Science Online.

33. X. L. Li, K. Chen, Y. Liu, Z. X. Wang, T. W. Wang, J. L. Zuo, Y. Z. Li, Y. Wang, J. S. Zhu, J. M. Liu, Y. Song, X. Z. You, Molecule-Based Ferroelectric Thin Films: Mononuclear Lanthanide Enantiomers Displaying Room-Temperature Ferroelectric and Dielectric Properties. Angew. Chem. Int. Edit. 46, 6820-6823 (2007); published online Epub2007/09/10 (10.1002/anie.200701802).

34. Y. Sui, D.-P. Li, C.-H. Li, X.-H. Zhou, T. Wu, X.-Z. You, Ionic Ferroelectrics Based on Nickel Schiff Base Complexes. Inorg. Chem. 49, 1286-1288 (2010); published online Epub2010/02/15 (10.1021/ic902136f).

35. S. Elisabeth, Piezoresponse force microscopy (PFM). J. Phys. D: Appl. Phys. 44, 464003 (2011).

36. M. S. Ivanov, V. A. Khomchenko, M. Salimian, T. Nikitin, S. Kopyl, A. M.

Buryakov, E. D. Mishina, F. Salehli, P. A. A. P. Marques, G. Goncalves, R. Fausto, J. A. Paixão, A. L. Kholkin, Self-assembled diphenylalanine peptide microtubes covered by reduced graphene oxide/spiky nickel nanocomposite: An integrated nanobiomaterial for multifunctional applications. Materials \& Design 142, 149-157 (2018); published online Epub2018/03/15/ (https://doi.org/10.1016/j.matdes.2018.01.018).

37. R. K. Vasudevan, N. Balke, P. Maksymovych, S. Jesse, S. V. Kalinin, Ferroelectric or non-ferroelectric: Why so many materials exhibit "ferroelectricity" on the nanoscale. App. Phys. Rev. 4, 021302 (2017)10.1063/1.4979015).

38. N. Balke, P. Maksymovych, S. Jesse, A. Herklotz, A. Tselev, C.-B. Eom, I. I. Kravchenko, P. Yu, S. V. Kalinin, Differentiating Ferroelectric and Nonferroelectric Electromechanical Effects with Scanning Probe Microscopy. ACS Nano 9, 6484-6492 (2015); published online Epub2015/06/23 (10.1021/acsnano.5b02227).

39. Y.-M. You, W.-Q. Liao, D. Zhao, H.-Y. Ye, Y. Zhang, Q. Zhou, X. Niu, J. Wang, P.F. Li, D.-W. Fu, Z. Wang, S. Gao, K. Yang, J.-M. Liu, J. Li, Y. Yan, R.-G. Xiong, An 
organic-inorganic perovskite ferroelectric with large piezoelectric response. Science 357, 306-309 (2017)10.1126/science.aai8535).

40. D.-W. Fu, H.-L. Cai, Y. Liu, Q. Ye, W. Zhang, Y. Zhang, X.-Y. Chen, G.

Giovannetti, M. Capone, J. Li, R.-G. Xiong, Diisopropylammonium Bromide Is a High-Temperature Molecular Ferroelectric Crystal. Science 339, 425-428 (2013).

41. H.-Y. Ye, Y.-Y. Tang, P.-F. Li, W.-Q. Liao, J.-X. Gao, X.-N. Hua, H. Cai, P.-P. Shi, Y.-M. You, R.-G. Xiong, Metal-free three-dimensional perovskite ferroelectrics. Science 361, 151 (2018)10.1126/science.aas9330).

42. K. Aizu, Possible Species of Ferromagnetic, Ferroelectric, and Ferroelastic Crystals. Phys. Rev. B 2, 754-772 (1970); published online Epub08/01/ (10.1103/PhysRevB.2.754).

43. P.-P. Shi, Y.-Y. Tang, P.-F. Li, W.-Q. Liao, Z.-X. Wang, Q. Ye, R.-G. Xiong, Symmetry breaking in molecular ferroelectrics. Chem. Soc. Rev. 45, 3811-3827 (2016)10.1039/C5CS00308C).

44. Y.-Y. Tang, P.-F. Li, W.-Q. Liao, P.-P. Shi, Y.-M. You, R.-G. Xiong, Multiaxial Molecular Ferroelectric Thin Films Bring Light to Practical Applications. J. Am. Chem. Soc. 140, 8051-8059 (2018); published online Epub2018/07/05 (10.1021/jacs.8b04600).

45. S. Xie, F. Ma, Y. Liu, J. Li, Multiferroic CoFe2O4-Pb(Zr0.52Ti0.48)O3 core-shell nanofibers and their magnetoelectric coupling. Nanoscale 3, 3152-3158 (2011)10.1039/C1NR10288E).

46. H. Miao, X. Zhou, S. Dong, H. Luo, F. Li, Magnetic-field-induced ferroelectric polarization reversal in magnetoelectric composites revealed by piezoresponse force microscopy. Nanoscale 6, 8515-8520 (2014)10.1039/C4NR01910E).

47. L. F. Henrichs, O. Cespedes, J. Bennett, J. Landers, S. Salamon, C. Heuser, T. Hansen, T. Helbig, O. Gutfleisch, D. C. Lupascu, H. Wende, W. Kleemann, A. J. Bell, Multiferroic Clusters: A New Perspective for Relaxor-Type Room-Temperature Multiferroics. Adv. Funct. Mater. 26, 2111-2121 (2016); published online Epub2016/04/01 (10.1002/adfm.201503335).

48. J. M. Caicedo, J. A. Zapata, M. E. Gómez, P. Prieto, Magnetoelectric coefficient in BiFeO3 compounds. J. Appl. Phys. 103, 07E306 (2008); published online Epub2008/04/01 (10.1063/1.2839276).

49. C. A. F. Vaz, J. Hoffman, C. H. Ahn, R. Ramesh, Magnetoelectric Coupling Effects in Multiferroic Complex Oxide Composite Structures. Adv. Mater. 22, 2900-2918 (2010); published online Epub2010/07/20 (10.1002/adma.200904326).

50. D. Lee, S. M. Yang, T. H. Kim, B. C. Jeon, Y. S. Kim, J.-G. Yoon, H. N. Lee, S. H. Baek, C. B. Eom, T. W. Noh, Multilevel Data Storage Memory Using Deterministic Polarization Control. Adv. Mater. 24, 402-406 (2012)10.1002/adma.201103679).

51. L. Bumagina, V. Krotov, B. Malkin, A. K. Khasanov, Magnetostriction in ionic rare earth paramagnets. Zh. Eksp. Teor. Fiz 80, 1543-1553 (1981).

Acknowledgments. We thank J. Haines for fruitful discussions on crystallography. F. Salles is acknowledged for discussion on the point charge model. Funding: The authors are grateful to University of Montpellier (UM), Centre National de la Recherche Scientifique (CNRS), Plateforme d'Analyse et de Caractérisation (PAC) et Institut Charles Gerhardt de Montpellier (ICGM), Fundação para a Ciência e a Tecnologia (FCT) Portugal (R\&D project No. PDTC/QUI-QUI/098098/2008-FCOMP-01-0124-FEDER-010785) and NoE FAME for financial support. M. S. I. is grateful to FCT for financial support through the project MATIS - Materiais e Tecnologias Industriais Sustentáveis (CENTRO-01-0145-FEDER-000014). V. A. K. is grateful to FCT for financial support through the FCT Investigator Programme (project IF/00819/2014/CP1223/CT0011). This work was partly supported by funds from FEDER (Programa Operacional Factores de Competitividade COMPETE) 
and from FCT under the projects CICECO - Aveiro Institute of Materials (UID/CTM/50011/2019) and UID/FIS/04564/2016. Access to TAIL-UC facility funded under QREN-Mais Centro project ICT_2009_02_012_1890 is gratefully acknowledged as well. Authors contributions: All authors contribute equally to this work. M. S. Ivanov supervised, conceived and planned the SPM experiments. V. A. Khomchenko conducted the PFM and ME discussions. M. S. Henriques and J. A. Paixao performed the single-crystal XRD characterization. L. D. Carlos and R. A. S. Ferreira measured the photoluminescence. B. Donnadieu resolved the crystal structures. E. Mamontova and J.-M. Thibaud performed the synthesis of the materials and standard characterizations. J. Rouquette and M. Beaudhuin measured the macroscopic dielectric and ferroelectric properties. D. Granier and J. Rouquette performed the single-crystal XRD characterization under magnetic field. J. Larionova and Y. Guari provided critical feedback and helped to the analysis and writing process of the manuscript. J. Long conceived the study, performed the magnetic characterization and wrote the manuscript with inputs from all authors. Competing interests: All authors declare that they have no competing interests. Data and materials availability: All data are available in the main text or the supplementary materials.

\section{Supplementary Materials:}

Materials and Methods

Figs. S1-S32

Tables S1-S11

References (52-85) 